\title{
Updates in Solving the Mystery of Alzheimer's Disease Pathology
}

\section{Regina $\mathrm{Au}^{*}$}

Principal, New Product Planning/Strategic Commercial Consultant BioMarketing Insight, Boston, Massachusetts, USA

\section{Introduction}

In a healthy brain, there are nerve cells or neurons (triangle figures) and synapses (the branches coming out of the nerve cells) as depicted in Figure 1. In Alzheimer's disease (AD), there are fewer nerve cells and synapses. Plaques are abnormal clusters of beta amyloid protein fragments that build up between nerve cells depicted as orange balls in Figure 1. Dead and dying nerve cells (black triangle figures) contain tangles, which are made up of twisted strands of a protein called Tau.

For years, investigators have believed that the pathology of Alzheimer's disease $(\mathrm{AD})$ was driven by the production and deposition of the $\beta$-amyloid peptide $(A \beta)$. But investigators have not been able to prove this theory as there is only a weak to nonexistent correlation between the amount of neuritic plaque pathology in the human brain and the degree of clinical dementia.

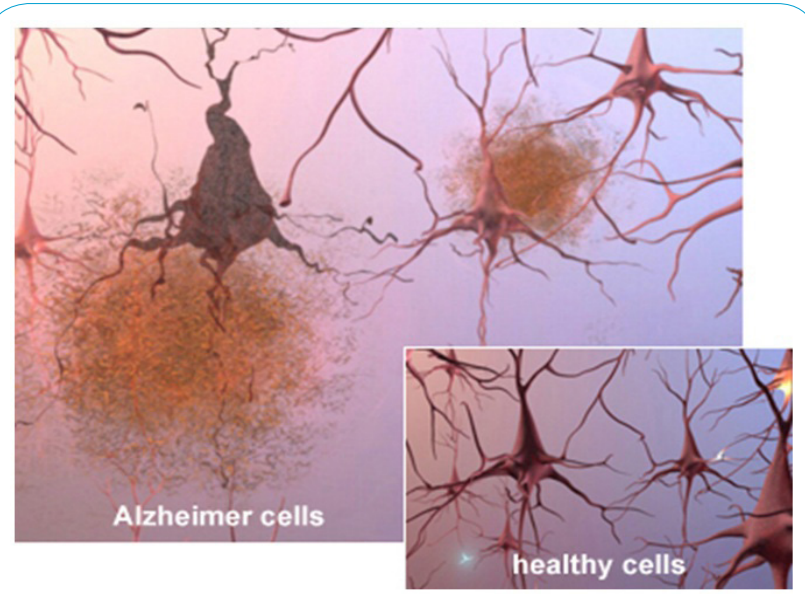

Figure 1: Inside a brain with Alzheimers; plagues are the orange cluster ball of beta amyloid protein near the nerve cell. Tangles causes a nerve cell to die and are located near the plague clusters.

Today, researcher have found substantial evidence to indicate that the solubility of $A \beta$, and the quantity of $A \beta$ in different pools, may be more closely related to disease state [1]. New imaging technologies such as positron emission tomography (PET) scan, including new amyloid imaging agents or radioactive drugs (tracers) that studies the metabolic activity or body function have made it possible to track amyloid pathology along with disease progression in living patients.

It is now believed that there are two hallmark pathologies required for a diagnosis of Alzheimer's disease (AD): (1) extracellular plaque deposits of the $\beta$-amyloid peptide $(A \beta)$; and (2) flame-shaped neurofibrillary tangles (NFTs) of the microtubule binding protein tau[1]. Early onset forms of AD are associated with genetic markers or mutations in either the precursor protein for $A \beta$ (the $\beta$-amyloid precursor protein, APP) or in presenilin-1 (PS1) or presenilin-2 (PS2). Despite this genetic evidence and the involvement of $A \beta$ in inducing synaptic dysfunction, disrupting neural connectivity, the amounts and distribution of $A \beta$ deposition have shown only a weak correlation to progressive and gradual decline in cognitive function.

\section{Publication History:}

Received: December 26, 2017

Accepted: June 04, 2018

Published: June 06, 2018

\section{Keywords:}

Alzheimer's disease, Nerve cells, Human brain, Amyloid pathology, Neural connectivity, Pathology

It has been generally accepted that there are a number of precursors or measurable biomarkers that need to occur before the brain develops AD. The updated proposed timeline of biomarker events that occur are depicted in Figure 2. The $\mathrm{x}$-axis is based on time rather than clinical stages because the time for each individual to develop cognitive impairment will vary. Each phase will be discussed in more detail regarding the pathological evidence to this hypothesis and the additional information required to further the science in discovering the pathology behind $\mathrm{AD}$.

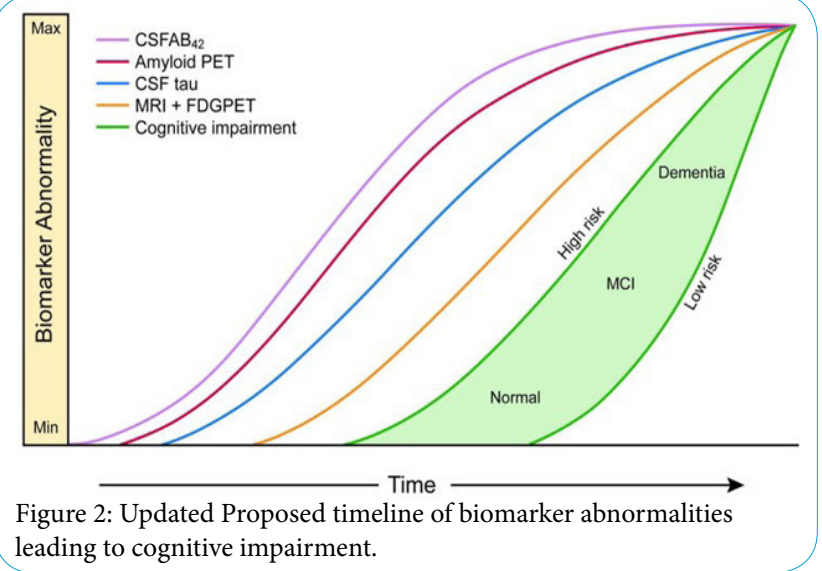

Phase 1: Cerebral Spinal Fluid ABeta ${ }_{42}\left(\mathrm{CSFAB}_{42}\right)$ and Interstitial Fluid (ISF) Clearance

\section{$\beta$-amyloid $(\mathrm{A} \beta)$ Clearance}

It was theorized that the pathology of Alzheimer's disease (AD) was driven by the over production and deposition of the $\beta$-amyloid peptide. AD classified as dementia can be divided into two groups: (1) early-onset $\mathrm{AD}$ (EOAD) and (2) sporadic or late-onset $\mathrm{AD}$ (LOAD). EOAD affects a minority of $\mathrm{AD}$ patients, whereas LOAD afflicts over $95 \%$ of patients with AD [3]. "Corresponding Author: Regina Au, Principal, New Product Planning/Strategic Commercial Consultant BioMarketing Insight, Boston, Massachusetts, USA; E-mail: regina@biomarketinginsight.com

Citation: Au R (2018) Updates in Solving the Mystery of Alzheimer's Disease Pathology. Int J Clin Pharmacol Pharmacother 3: 138. doi: https://doi. org/10.15344/2018/2456-3501/138

Copyright: (C) $2018 \mathrm{Au}$. This is an open-access article distributed under the terms of the Creative Commons Attribution License, which permits unrestricted use, distribution, and reproduction in any medium, provided the original author and source are credited. 
Citation: Au R (2018) Updates in Solving the Mystery of Alzheimer's Disease Pathology. Int J Clin Pharmacol Pharmacother 3: 138. doi: https://oi. $\operatorname{org} / 10.15344 / 2018 / 2456-3501 / 138$

Page 2 of 4

Both EOAD and LOAD are characterized by excessive accumulation of toxic forms of $A \beta$, which now has been hypothesized to be due to the malfunction in the clearance of $A \beta$ protein rather than the over production that leads to $A \beta$ accumulation. Emerging evidence also suggests that $A \beta$ clearance is impaired in both early-onset and late-onset forms of $\mathrm{AD}$ as $\mathrm{A} \beta$ proteins can be detected and measured in the CSF and ISF [2]

Soluble $A \beta$ can be removed from the brain by various clearance systems, including enzymatic degradation and cellular uptake, transport across the blood-brain barrier (BBB) and bloodcerebrospinal fluid barrier (BCSFB), interstitial fluid (ISF) bulk flow, and cerebrospinal fluid (CSF) absorption into the circulatory and lymphatic systems [3].

Mouse studies conducted in the early 2000 s, demonstrated that the majority $(75 \%)$ of extracellular $A \beta(\mathrm{eA} \beta)$ is cleared by the $\mathrm{BBB}$, with only a minority (10\%) being cleared by ISF bulk flow. However, recent imaging studies suggested that ISF bulk flow-facilitated by astroglial aquaporin-4 (AQP4) channels and the glymphatic (glial + lymphatic) system-contributes to a larger portion of eA $\beta$ clearance than previously thought [3].

The presence of neuritic plaques that are composed for the most part of highly insoluble $A \beta$ in the brain parenchyma is required for a diagnosis of $\mathrm{AD}$ [1]. Deposits of tau protein are also present, although they are also found in a number of less common neurodegenerative diseases, notably in the absence of neuritic plaques.

\section{Phase 2: Beta-Amyloid Plaque}

Beta Amyloid is derived from a larger protein found in the fatty membrane surrounding nerve cells and are chemically sticky and can gradually builds up to form plague [4]. See Figure 3. It is believed that small pieces of $A \beta$ protein may block cell-to-cell signaling at synapses or activate the immune system that promotes inflammation and destroys cells.

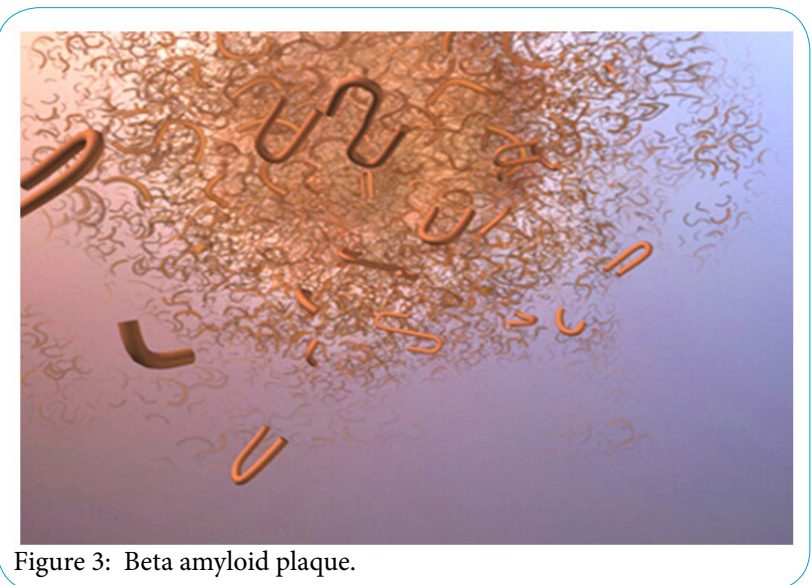

Scientists know that the accumulation of $A \beta$ plague is toxic and the most logical course of action is to eliminate or decrease the amount of plaque to prevent or halt the progression of $\mathrm{AD}$. A number of pharmaceutical companies have conducted clinical trials to decrease $\mathrm{A} \beta$ plague, but failure to demonstrate the slowing progression of $\mathrm{AD}$. Why some people have a massive amount of plaque that does not progress to the development of dementia is still a mystery. All we know, is that soluble $A \beta$ can be cleared from the brain and insoluble $\mathrm{A} \beta$ forms plaques.
Here are a couple of theories regarding this matter: [1]

1. While the minimum or maximum amount of $A \beta$ deposition could contribute to the onset of the disease it's probably highly individualized similar to personalized medicine. It has been theorized that $A \beta$ exerts its major effects early by triggering a cascade of events that, once begun, proceed independently of $\mathrm{A} \beta$ being removed. It is unclear to the exact mechanism of this theory except to surmise that the continued accumulation of misfolded hyperphosphorylated tau, leads to further neuron loss.

2. Another possible explanation is that specific form or forms of $A \beta$ are responsible for the massive neuronal death that accompanies this disease.

Phase 3: Tau clearance and tau-mediated neuronal injury and dysfunction.

\section{Tau clearance}

This is a new biomarker abnormality [2]. Tau is an intracellular neuronal protein that stabilizes axons and microtubules. Intracellular tau (i-tau) can undergo two transformations that are relevant to its clearance: modification and release [3]. Tau modification is regulated by phosphorylation. In $\mathrm{AD}$, i-tau is hyperphosphorylated, which induces the formation of insoluble Neurofibrillary Tangles (NFTs) that cannot readily be cleared, and can also be neurotoxic. Neuronal activation, neuronal death and increased i-tau concentration or aggregation triggers the release of $i$-tau into the extracellular space, leading to elevated CSF tau levels.

The clearance of Tau from the brain is not well understood but it appears to be less complex than beta amyloid since there seems to be no evidence that it is cleared by the BBB, except after brain injury, when BBB permeability is temporarily increased. Tau is hypothesized to be cleared from the brain primarily by degradation, ISF bulk flow, and CSF absorption clearance [3].

Studies at Children's Hospital in Boston, Massachusetts have recently discovered that there are two types of Tau proteins, soluble tau (good tau) and insoluble tau (possibly hyperphosphorylated tau). The significant of both has not been determined, yet one can guess that the soluble tau gets cleared and the insoluble tau probably induces the formation of insoluble NFTs which in turn lead to nerve cell death.

The pathological accumulation of $A \beta$ and tau proteins in the brain can be inferred by analyzing their levels in the CSF. Specifically, $\mathrm{A} \beta$ accumulation into extracellular plaques is marked by decreased CSF levels of $A \beta 1_{42}$, and tau accumulation into NFTs is marked by increased CSF levels of total tau and hyperphosphorylated tau [3]. In addition, PET can be used to assess A $\beta$ brain accumulation directly, and PET for tau is currently under investigation.

Buchave, et al. have found that $\mathrm{A} \beta_{42}$ was fully abnormal 5-10 years or more prior to a dementia diagnosis [5]. In contrast, both CSF $\mathrm{t}$-tau and p-tau became progressively more abnormal as the time to diagnosis of dementia decreased. P-tau and t-tau behaved identically over time.

\section{tau- mediated neuronal injury and dysfunction}

In healthy areas, tau proteins control the microtubule stability through isoforms and phosphorylation. Tau helps to keep the transport system in tack like a railroad track. Hyperphosphorylation 
Citation: Au R (2018) Updates in Solving the Mystery of Alzheimer's Disease Pathology. Int J Clin Pharmacol Pharmacother 3: 138. doi: https://oi. $\operatorname{org} / 10.15344 / 2018 / 2456-3501 / 138$

Page 3 of 4

of tau proteins can cause the helical and straight filaments to twist or tangle (NFTs). Tangles form inside dying nerve cells and contribute to the pathology of Alzheimer's disease [6]. The twisted strands of tau (hyperphosphorylated) essentially destroys the transport system so that nutrients and other essential supplies can no longer move through the cells, which eventually die [4]. See Figure 4.

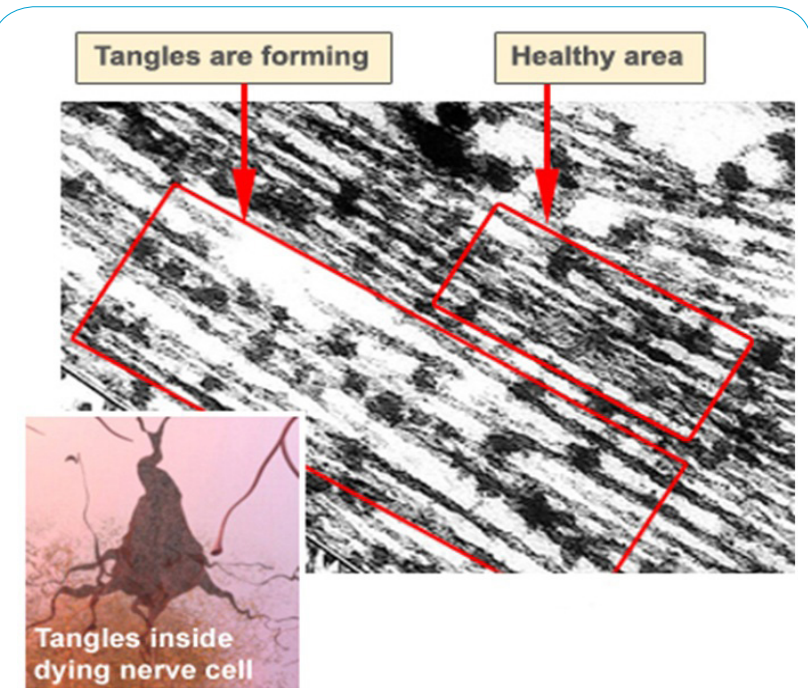

Figure 4: The healthy area outlined in red is microtubules regulated by tau. The unhealthy area is hyperphosphorylated tau that lead to tangles or disintegration of the microtubules.

Though most people develop some plaques and tangles as they age, those with Alzheimer's tend to have a tremendous amount of both. The plaques and tangles tend to form in a predictable pattern, beginning in areas important in learning and memory and then spreading to other regions [6].

When a brain affected by Alzheimer's disease is examined, all six isoforms of tau are often found hyperphosphorylated in paired helical filaments [6]. Deposits of abnormal aggregates enriched with tau isoforms have also been reported in some other neurodegenerative diseases.

\section{Phase 4: Brain Structure, Memory, Clinical Function}

In the Clifford, et al. study, they hypothesis that after the aberrant changes of $A \beta$ and tau, one will see structural changes of hypometabolism on fluorescent fluorodeoxyglucose (FDG)- PET and atrophy of the hippocampal on structural MRI followed by clinical symptoms [2].

The pathology as to the events that need to occur and the exact order for these three phases progressing to cognitive impairment is still unclear as there is contradictory evidence to the current model of $A \beta$ pathophysiology occuring first, then tau related neurodegeneration in the elderly and young mutation carriers. The contradictory evidence from autopsy data in young individuals indicating that $\mathrm{AD}$-like tauopathy precedes $\mathrm{A} \beta$ deposition $[2,7]$ One possible explanation according Jack et al. is that tauopathy and $\mathrm{A} \beta$ opathy arises independently assuming that $\mathrm{AD}$ pathophysiology lies undetected by current diagnostics tools for every in-vivo $\mathrm{AD}$ biomarker. This also assumes that subcortical tauopathy is the first $\mathrm{AD}$ pathophysiology to appear in some individuals, detectable only by immunostaining methods. Since tauopathy alone does not lead to $\mathrm{AD}$ dementia, it maybe the initiation of A $\beta$ opathy that leads to these cascade of events.
There are a number of hypothesis of the events following tau related neurodegeneration in the elderly and young mutation carriers:

1. Neuroinflammation, a relatively need field can be divided into two subgroups: [8]

1. Neuroinflammation-where pieces of $A \beta$ protein activate the immune system that promotes inflammation and possible destroys brain cells through the innate immune system.

2. Neuroinflammation that does not reproduce the classic characteristics of inflammation in the periphery, but may induce neurodegenerative events; including plaque formation, dystrophic neurite growth, and excessive tau phosphorylation.

2. That specific form or forms of $A \beta$ are responsible for the massive neuronal death that accompanies this disease [1]. There are currently no imaging or scientific tools available to confirm this theory in distinguishing the difference between what maybe disease-related $A \beta$ from less relevant forms thus, weakening the correlation with clinical stages.

3. Neuron-glia and neural-immune interactions in the role of synaptic pruning and the up regulations of synaptic pruning that leads to significant synaptic loss. Beth Stevens and her team at Children's Hospital in Boston, Massachusetts are investigating this hypothesis through their work with $\mathrm{Clq}$, the initiating protein of the classical complement cascade, that tags unwanted cells and debris for elimination in the developing visual system [9]. They found that C1q becomes abnormally upregulated and hone in to synapses in the early stages of glaucoma. There may be a similar mechanism for neurodegenerative disease which they are currently pursuing.

4. It has already been shown that those who have the APOE4 mutation, a risk factor for AD, that APOE4 has an effect on glucose metabolism that temporally precedes amyloid deposition [2].

A recent study conducted by the National Institute on Aging (NIA), National Institutes of Health, demonstrated that abnormalities in glycolysis or the breakdown of glucose in the brain, is connected to the severity of Alzheimer's Pathology [10]. Reduction in brain glycolysis produces higher levels of brain glucose which correlated to more severe plaques and tangles found in the brains of people with AD. Severer reduction in brain glycolysis were also related to the expression of symptoms of $\mathrm{AD}$, such as problems with memory.

Scientists found that GLUT3, a glucose transporter protein, in neurons, the levels were lower in brains with Alzheimer's pathology compared to normal brains [10]. The lower the levels of GLUT3 the greater the severity of tangles and plaques. They also found that when following blood glucose levels in patients until death, an increase in blood glucose correlated with an increase in brain glucose at death.

\section{Conclusion}

The pathology of the brain is very complex, and it is only after decades of research and the invention of advanced imaging equipment and other scientific tools have researchers begun to unravel the mystery of the brain. Scientists and physicians have agreed upon one thing; in order to prevent or slow the progression of this disease, one needs to identify biomarkers 10 - 20 years before patients show classical symptoms of dementia, more specifically biomarkers that precede $A \beta$. 
It is a consensus that both $A \beta$ and tau proteins play an important role in the pathology $\mathrm{AD}$, but the debate remains on whether reducing the aggregation of both insoluble $A \beta$ and tau proteins, halts or reduces the progression of $\mathrm{AD}$ or whether there is a threshold of tau opathy and $A \beta$ opathy that sets off a cascade of events that is irreversible and independent to the amount of aggregation of $A \beta$ and tau. Scientist know that soluble $A \beta$ and tau is cleared from the brain, and that insoluble $A \beta$ leads to plaque and hyperphosphorylated tau, possibly insoluable tau induces the formation of tangles.

However, some recent studies have supported the evidence that soluble $A \beta(s A \beta)$ oligomers are an early trigger of synpatic damage and cognitive impairment in AD. There seems to be a strong correlations between $s A \beta$ levels and the severity of neuropathological changes in $\mathrm{AD}$, where sA $\beta$ has the ability to cause synaptic failure and cognitive function disruption [11].

Some Industry scientists believe that tangles are comprised of soluble and insoluble tau depending on the stage of the tangle development in the nerve cells since tau is needed to stabilize the microtubules that act as a path of communications and nutrients for nerve cells.

We know this disease is not a linear progression and that there may be a host of other factors such as impaired glycolysis, specific forms of disease related $A \beta$, specific isoforms of tau, individual thresholds of tau opathy and $A \beta$ opathy and neuroinflammation, non-characteristic of inflammation in the periphery, where one to all may possibly be involved in this cascade of events leading to dementia and then AD. Other contributing events could include comorbidities, environmental factors, our microbiome, even pathology or biomarkers that scientists have yet to discover, could all influence in varying degrees just like personalized medicine whether one would develop AD. Solving this puzzle is very similar to solving a Rubix Cube for the first time.

Which theory is correct and which is incorrect when there is contradictory evidence? Only time will tell. But we need to investigate it all until we get the right answers in fully understanding the pathology of $\mathrm{AD}$ and other neurodegenerative disease.

\section{Competing Interests}

The author declare no competing interests.

\section{References}

1. Murphy MP, LeVine H (2010) Alzheimer's Disease and the $\beta$-Amyloid Peptide. J Alzheimers Dis. 2010 Jan; 19: 311.

2. Jack CR Jr, Knopman DS, Jagust WJ, Petersen RC, Weiner MW, et al (2013) Update on hypothetical model of Alzheimer's disease biomarkers, Lancet Neurol. 12: 207-216.

3. Tarasoff-Conway JM, Carare RO, Osorio RS, Glodzik L, Butler T, et al (2015) Clearance systems in the brain-implications for Alzheimer disease, Nat Rev Neurol. 11: 457-470.

4. Alzheimer's Association (2011) Brain Tour

5. Buchhave P, Minthon L, Zetterberg H, Wallin AK, Blennow K, et al. (2012) Cerebrospinal Fluid Levels of $\beta$-Amyloid 1-42, but Not of Tau, Are Fully Changed Already 5 to 10 Years Before the Onset of Alzheimer Dementia. Arch Gen Psychiatry 69: 98-106.

6. New Medical Life Sciences (2014) What are Tau Proteins?
7. Braak H, Del Tredici K (2011) The pathological process underlying Alzheimer's disease in individuals under thirty. Acta Neuropathol 121: 171181.

8. Streit WJ, Mrak RE, Griffin WS (2004) Microglia and neuroinflammation: a pathological perspective, Journal of Neuroinflammation 1: 14 .

9. Stevens B Lab, FM Kirby Neurobiology Center, Research Associate in Neurology, Children's Hospital and Harvard Medical School, Boston, Massachusetts.

10. Eurekalert, Global Source for Science News (2017) Higher brain glucose levels may mean more severe Alzheimer's, Press Release

11. Li JJ, Dolios G, Wang R, Liao FF (2014) Soluble Beta-Amyloid Peptides, but Not Insoluble Fibrils, Have Specific Effects on Neuronal MicroRNA Expression, PLoS One 9: e90770 